\title{
EVAPOTRANSPIRAÇÃO DE REFERÊNCIA BASEADA EM MÉTODOS EMPÍRICOS EM BACIA EXPERIMENTAL NO ESTADO DE PERNAMBUCO - BRASIL
}

\author{
ADA RAVANA COSTA MOURA, SUZANA Ma GICO LIMA MONTENEGRO, ANTÔNIO CELSO \\ DANTAS ANTONINO, JOSÉ ROBERTO GONÇALVES DE AZEVEDO, BERNARDO BARBOSA \\ DA SILVA, LEIDJANE M ${ }^{a}$ MACIEL DE OLIVEIRA
}

\author{
Universidade Federal de Pernambuco (UFPE), Departamento de Engenharia Civil, Recife, PE, Brasil \\ adaravana@gmail.com,suzanam@ufpe.br, acda@ufpe.br,jrga@ufpe.br, bbdasilva.ufpe@gmail.com, \\ leidjaneoliveira@hotmail.com
}

Recebido Dezembro de 2011 - Aceito Setembro de 2012

\begin{abstract}
RESUMO
O estudo da evapotranspiração é importante para o planejamento agrícola, principalmente em regiões caracterizadas por grandes variabilidades espacial e temporal da precipitação, como nas áreas áridas e semiáridas do Nordeste do Brasil. Embora existam no Brasil duas grandes redes de observação meteorológica (INMET e CPTEC), ainda há muitas regiões não cobertas pelas mesmas, evidenciando a necessidade de métodos de cômputo da evapotranspiração baseados em poucos elementos meteorológicos. Considerando esta necessidade, este trabalho objetivou determinar a evapotranspiração de referência (ETo) por métodos indiretos em várias escalas de tempo na bacia experimental do riacho Gameleira, Vitória de Santo Antão, PE. Os métodos indiretos foram avaliados tendo como padrão o método Penman-Monteith FAO-56. Dentre os métodos baseados na temperatura do ar, Hargreaves-Samani (1985) apresentou desempenho "ótimo" nas escalas pentadal a mensal, seguido de Blaney-Criddle com desempenho "muito bom". Já os métodos de Camargo, Thornthwaite modificado e Thornthwaite apresentaram variações de desempenho de "mau a muito bom". Dos métodos baseados na radiação solar, o melhor desempenho foi o método da Radiação Solar, seguido de Makkink e Jensen-Haise. Diferentemente, os métodos baseados em modelagem estatística não estimaram a ETo com boa precisão, resultando em baixos coeficientes de determinação e desempenho.

Palavras-chave: Penman-Monteith, métodos indiretos, semiárido
\end{abstract}

\begin{abstract}
EVAPOTRANSPIRATION REFERENCE BASED ON EMPIRICAL METHODS IN A EXPERIMENTAL BASIN AT THE STATE OF PERNAMBUCO - BRAZIL

The study of evapotranspiration is important for agricultural planning, especially in regions characterized by high spatial and temporal variability of precipitation, as in arid and semiarid regions of northeastern Brazil. Although in Brazil there are two large meteorological observation networks (INMET and CPTEC), there are still many uncovered areas, suggesting the need for methods of calculating evapotranspiration based on few meteorological elements. Considering this need, this study aimed to determine the reference evapotranspiration (ETo) by indirect methods in various time scales in the experimental watershed of the creek Gameleira, Vitoria de Santo Antão, PE. Indirect methods have been evaluated considering the Penman-Monteith FAO-56 as the standard method. Among the methods based on air temperature, the Hargreaves-Samani (1985) presented an "optimal" performance at pentadal to monthly scales, followed by the Blaney-Criddle with performance "very good". The Campbell, Thornthwaite and the modified Thornthwaite methods showed variations of performance from "bad to very good". Among the solar radiation based methods, the best performance was the solar radiation method, followed by Makkink and Jensen-Haise. The methods based on statistical modeling did not estimate ETo with good accuracy, resulting in low determination and performance coefficients values.
\end{abstract}

Keywords: Penman-Monteith, indirect methods, semi-arid 


\section{INTRODUÇÃO}

O estudo da evapotranspiração é importante para o planejamento agrícola, sendo cada vez maior a exigência de informações sobre as necessidades hídricas das culturas para o planejamento regional e preliminar de projetos. Este estudo se torna mais importante em regiões caracterizadas pela irregularidade espacial e temporal da precipitação, e pela escassez de reservas naturais de água, como nas áreas áridas e semiáridas do Nordeste do Brasil.

Estudos recentes sobre o aquecimento global e os cenários futuros da agricultura brasileira apontam que o conhecimento da demanda hídrica das culturas, diante das mudanças climáticas, influenciará na definição das estratégias regionais de manejo da água (Assad e Pinto, 2008). Aliado a estes fatores, a crescente demanda pelo uso da água e os conflitos pela sua utilização, devido aos múltiplos usos, mostram a importância de se ter informações precisas e seguras dos diversos tipos de demanda, dentre eles o da evapotranspiração.

A evapotranspiração de cultura é fundamental para o adequado dimensionamento de projetos e no manejo de irrigação, pois representa a quantidade de água a ser reposta ao solo para manter o crescimento e a produção em condições ideais (Pereira et al., 1997). Sua determinação pode ser dada pelo produto entre a evapotranspiração de referência (ETo) e o coeficiente de cultura $(\mathrm{Kc})$, que varia de acordo com o tipo de cultura e seu estádio fenológico.

Existem diversos métodos para determinação da evapotranspiração, sejam eles diretos ou indiretos. Dentre os métodos diretos destaca-se a utilização dos lisímetros e do balanço hídrico no solo. No caso dos métodos indiretos, destaca-se o modelo de Penman-Monteith, recomendado pela FAO (Food and Agriculture Organization) no boletim de Irrigação e Drenagem no 56 (FAO-56), como padrão para determinação da evapotranspiração de referência (Allen et al., 1998).

Conceição e Mandelli (2005) avaliaram os métodos de Hargreaves-Samani, Camargo, Thornthwaite e Thornthwaite modificado, baseados na temperatura do ar e os métodos de Makkink e radiação, baseados na radiação solar, comparados com o método de Penman-Monteith FAO-56, utilizando um coeficiente de desempenho, resultante da multiplicação do coeficiente de correlação e de exatidão. Os autores concluíram que os métodos baseados na radiação apresentaram melhor desempenho do que os métodos baseados na temperatura do ar. Os resultados mostraram um bom desempenho do método de Thornthwaite modificado, em relação ao método de Thornthwaite original. Este resultado, segundo os autores, foi decorrente da utilização da temperatura efetiva, pois esta representa melhor as condições meteorológicas do período diurno.
Turco et al. (2008) avaliaram os erros da estimativa da ETo em função da combinação dos erros das variáveis que compõem as equações de Makkink, Hargreaves, tanque Classe A, Radiação solar e Penman-Monteith FAO-56. Os métodos foram comparados com Penman-Monteith FAO-56 e os resultados apontaram que o melhor ajuste foi do método de Hargreaves. Com a metodologia de análise dos dados aplicada, não foi estabelecida relação linear entre os demais métodos, inviabilizando a comparação destes com PenmanMonteith.

Back (2008), em estudo realizado em Urussanga-SC, comparou as estimativas da ETo obtidas através de nove equações empíricas, baseadas na temperatura do ar, com o método de Penman-Monteith, nas escalas de tempo decendial e mensal. Dentre as equações utilizadas, destacam-se as equações de Thornthwaite, Thornthwaite modificada, Camargo, Hargraves-Samani e Blaney-Criddle.

Fernandes et al. (2012) calibraram o modelo de Hargreaves para estimar a ETo diária por meio de coeficientes empíricos de maneira local e regional, os autores concluíram que o estudo realizado pode ser uma alternativa onde a disponibilidade de dados climáticos é limitada.

Para utilização destes métodos é importante dispor de uma rede de monitoramento de dados meteorológicos (temperatura e umidade do ar, radiação solar, velocidade do vento e precipitação), de forma que se mantenha um banco de dados de referência com informações que forneçam subsídios para a avaliação dos estudos e projetos voltados à determinação da evapotranspiração. Embora existam no Brasil duas grandes redes (Instituto Nacional de Meteorologia - INMET e Centro de Previsão e Estudos Climáticos do Instituto Nacional de Pesquisas Espaciais - CPTEC/INPE) de observação meteorológica, ainda há muitas regiões produtoras agrícolas não cobertas pelas mesmas, o que evidencia a necessidade do uso de métodos de cômputo da evapotranspiração baseado em poucos elementos meteorológicos. Ademais, a existência de falhas em alguns elementos meteorológicos, ou a qualidade inadequada, muitas vezes impedem o uso do método padrão de Penman-Monteith. Fica evidente, diante do cenário apresentado, a importância do estudo da evapotranspiração e, consequentemente, da definição e uso de métodos precisos para sua estimativa.

Desta forma, este trabalho tem como objetivo geral avaliar as estimativas da evapotranspiração de referência (ETo) na bacia experimental do riacho Gameleira em Pernambuco, utilizando métodos baseados na temperatura do ar, radiação solar e em modelagem estatística. Como objetivos específicos têm-se o emprego destes métodos em diferentes escalas de tempo, tomando-se como padrão o método de Penman-Monteith (Allen et al., 1998). 


\section{MATERIAL E MÉTODOS}

Este estudo foi realizado na bacia do riacho Gameleira, localizada no município de Vitória de Santo Antão - PE, parte nordeste da Mesorregião Geográfica do Agreste do estado de Pernambuco, nas coordenadas $35^{\circ} 17^{\prime} \mathrm{E}, 8^{\circ} 04^{\prime} \mathrm{S}$ e $35^{\circ} 20^{\prime} \mathrm{E}$, $8^{\circ} 06^{\prime} \mathrm{S}$. Esta microbacia está inserida na bacia do rio Tapacurá, que por sua vez é uma sub-bacia do rio Capibaribe (Figura 1).

A microbacia do riacho Gameleira possui área de drenagem de $17 \mathrm{~km}^{2}$ e situa-se em área de transição zona da mata e o semiárido. O clima dominante da área é o As', clima quente e úmido, do tipo pseudo tropical segundo a classificação de Köeppen (Braga, 2001). O período chuvoso é entre março e julho, quando ocorrem $68 \%$ da precipitação anual. A precipitação anual média é de $1.047 \mathrm{~mm}$, a temperatura do ar mensal média entre $23,7^{\circ} \mathrm{C}$ (agosto) e $27,0^{\circ} \mathrm{C}$ (março), enquanto a umidade relativa do ar, durante os meses de março a julho, é superior a 70\% (Araújo Filho et al., 2007).

A microbacia do riacho Gameleira dispõe de uma rede de monitoramento de dados composta por cinco estações, implantadas pelo projeto "Implantação de bacias experimentais do semiárido - IBESA", inserido na Rede de Hidrologia do Semiárido - REHISA. Dentre estas, destaca-se a estação climatológica ( $8^{\circ} 6^{\prime} \mathrm{S}$ e $35^{\circ} 17^{\prime} \mathrm{E}$ ), onde são monitorados os seguintes elementos: precipitação, temperatura do ar, umidade relativa, radiação solar, temperatura do solo e velocidade e direção do vento. Os dados desta estação foram utilizados para o cálculo da evapotranspiração de referência através de 11 métodos indiretos, no período de janeiro de 2004 a abril de 2009, em diferentes escalas de tempo, tomando-se como estimativa padrão o método de Penman-Monteith FAO-56 (Allen et al., 1998).

Os métodos foram avaliados na escala de tempo diária, quinquidial, decendial, quinzenal e mensal, sendo eles: a) Método combinado: Penman-Monteith FAO-56 (padrão); b) Métodos baseados na temperatura do ar: Thornthwaite,

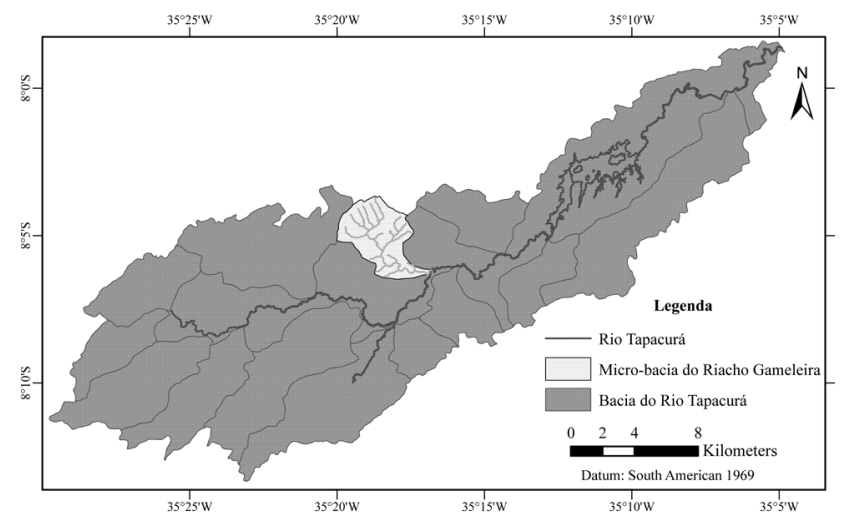

Figura 1 - Localização da bacia do riacho Gameleira inserida na bacia do rio Tapacurá.

Fonte: Moura (2009)
Blaney-Criddle, Camargo, Hargreaves-Samani e Thornthwaite modificado; c) Métodos baseados na radiação solar: Makkink, Jensen-Haise e radiação solar; d) Métodos baseados em modelagem estatística: Araújo Filho e Cirilo (1994) e Oliveira et al. (2007). O método de Araújo Filho e Cirilo (1994) foi avaliado apenas na escala de tempo mensal.

\section{Método de Penman-Monteith FAO-56}

O modelo de Penman-Monteith FAO-56 (Allen et al., 1998), obtido através de diversas parametrizações, é dada por:

$$
\mathrm{ETo}_{(\mathrm{PM}-56)}=\frac{0,408 \Delta\left(\mathrm{R}_{\mathrm{n}}-\mathrm{G}\right)+\gamma \frac{900}{\mathrm{~T}+273} \mathrm{u}_{2}\left(\mathrm{e}_{\mathrm{s}}-\mathrm{e}_{\mathrm{a}}\right)}{\Delta+\gamma\left(1+0,34 \mathrm{u}_{2}\right)}
$$

em que, $\mathrm{ET}_{\mathrm{O}(\mathrm{PM}-56)}$ é a evapotranspiração de referência estimada pelo método de Penman-Monteith FAO-56 ( $\left.\mathrm{mm} \mathrm{d}^{-1}\right) ; \mathrm{R}_{\mathrm{n}}$ é o saldo radiação a superfície $\left(\mathrm{MJ} \mathrm{m}^{2} \mathrm{~d}^{-1}\right)$; $\mathrm{G}$ é o fluxo de calor no solo $\left(\mathrm{MJ} \mathrm{m}^{2} \mathrm{~d}^{1}\right)$; $\mathrm{T}$ é a temperatura do ar média $\left({ }^{\circ} \mathrm{C}\right) ; \mathrm{u}_{2}$ é a velocidade do vento a $2 \mathrm{~m}$ de altura $\left(\mathrm{m} \mathrm{s}^{-1}\right)$; e é a pressão de vapor de saturação $(\mathrm{kPa})$; $\mathrm{e}_{\mathrm{a}}$ é a pressão real de vapor $(\mathrm{kPa}) ; \Delta$ é a tangente à curva da pressão de saturação do vapor d'água $\left(\mathrm{kPa}^{\circ} \mathrm{C}^{-1}\right)$ e $\gamma$ é o coeficiente psicrométrico $\left(0,0666 \mathrm{kPa}^{\circ} \mathrm{C}^{-1}\right)$, que foram obtidos segundo os procedimentos propostos no Boletim N ${ }^{\circ} 56$ da FAO (Allen et al., 1998) e quando pertinente o uso das recomendações para situações de escassez de dados.

\section{Método de Thornthwaite (1948)}

$$
\begin{aligned}
& \mathrm{ETo}_{(\mathrm{Th})}=16\left(10 \frac{\mathrm{T}_{\mathrm{i}}}{\mathrm{I}}\right)^{\mathrm{a}}, 0{ }^{\circ} \mathrm{C} \leq \mathrm{T}_{\mathrm{i}} \leq 26{ }^{\circ} \mathrm{C} \\
& \mathrm{ETo}_{(\mathrm{Th})}=-415,85+32,24 \mathrm{~T}_{\mathrm{i}}-0,43 \mathrm{~T}_{\mathrm{i}}^{2}, \quad \mathrm{~T}_{\mathrm{i}}>26^{\circ} \mathrm{C}
\end{aligned}
$$

em que, $\mathrm{ETo}_{(\mathrm{Th})}$ é a evapotranspiração de referência $\left(\mathrm{mm} \mathrm{mês}^{-1}\right)$, $\mathrm{T}_{\mathrm{i}}$ é a temperatura do ar média mensal $\left({ }^{\circ} \mathrm{C}\right)$ e I é o índice térmico imposto pelo regime climático local, calculado por:

$$
\begin{aligned}
& \mathrm{I}=\sum_{\mathrm{n}=1}^{12}\left(0,2 \mathrm{~T}_{\mathrm{i}}\right)^{1,514}, \mathrm{~T} \geq 0{ }^{\circ} \mathrm{C} \\
& \mathrm{a}=6,75 \cdot 10^{-7} \mathrm{I}^{3}-7,71 \cdot 10^{-5} \mathrm{I}^{2}+1,7912 \cdot 10^{-2} \mathrm{I}+0,49239
\end{aligned}
$$

em que, i representa o mês do ano $(\mathrm{i}=1$, janeiro; ...; $\mathrm{i}=12$, dezembro).

As Equações 2 e 3 fornecem a evapotranspiração para a condição padrão de meses com 30 dias e dias com 12 horas de brilho solar. Para obter a evapotranspiração diária, multiplica-se o valor obtido através das Equações 2 ou 3 pelo seguinte fator de correção (Pereira et al., 1997):

$$
\mathrm{C}=\frac{\mathrm{N}}{12} \cdot \frac{1}{30}
$$

em que, $\mathrm{N}$ é o fotoperíodo (h) de determinado dia. 
O fotoperíodo é calculado em função da latitude do local e da declinação solar, através da seguinte equação (VarejãoSilva, 2006):

$$
\mathrm{N}=\frac{2}{15} \omega_{\mathrm{s}}
$$

em que, $\mathrm{N}$ é o fotoperíodo (h) e $\omega_{\mathrm{s}}$ é o ângulo horário relativo ao nascer ou ao pôr do sol.

\subsection{Método de Thornthwaite modificado}

Camargo et al. (1999) ajustaram a equação original de Thornthwaite (1948) com o uso da temperatura efetiva $\left(\mathrm{T}_{\mathrm{ef}}\right)$, em função da temperatura do ar média e da amplitude diária de temperatura do ar, dada por:

$$
\mathrm{T}_{\mathrm{ef}}=\mathrm{k}\left(\mathrm{T}_{\mathrm{i}}+\mathrm{A}\right)
$$

onde, A é a amplitude térmica média diária.

A temperatura média e a amplitude são dadas por Camargo et al.(1999) como:

$$
\begin{aligned}
& \mathrm{T}=\left(\frac{\mathrm{T}_{\text {max }}+\mathrm{T}_{\min }}{2}\right) \\
& \mathrm{A}=\left(\mathrm{T}_{\text {max }}-\mathrm{T}_{\text {min }}\right)
\end{aligned}
$$

em que, $T_{\max }$ e $T_{\min }$ são a média mensal das temperaturas máxima e mínima $\left({ }^{\circ} \mathrm{C}\right)$, respectivamente.

Substituindo as Equações 9 e 10 na Equação 8, obtém-se a seguinte equação simplificada:

$$
\mathrm{T}_{\text {ef }}=0,5 \mathrm{k}\left(3 \mathrm{~T}_{\max }-\mathrm{T}_{\text {min }}\right)
$$

onde, $\mathrm{k}=0,72$ (Camargo et al., 1999) e $\mathrm{k}=0,69$ (Pereira e Pruitt, 2004).

Para determinar a evapotranspiração através do método de Thornthwaite modificado, utiliza-se a temperatura efetiva em substituição à temperatura média nas Equações 2 e 3.

\subsection{Método de Hargreaves-Samani}

$$
\mathrm{ETo}_{(\mathrm{HS})}=0,0023 \cdot \mathrm{R}_{\mathrm{T}} \cdot \sqrt{\mathrm{T}_{\text {max }}-\mathrm{T}_{\text {min }}} \cdot(\mathrm{T}+17,8)
$$

em que, $\mathrm{ETo}_{(\mathrm{HS})}$ é a evapotranspiração de referência $\left(\mathrm{mm} \mathrm{d}^{-1}\right)$ estimada pelo método de Hargreaves e Samani (1985); $\mathrm{R}_{\mathrm{T}}$ é a radiação solar extraterrestre $\left(\mathrm{mm} \mathrm{d}^{-1}\right)$.

\subsection{Método de Blaney-Criddle}

$$
\begin{aligned}
& \mathrm{ETo}_{(\mathrm{BC})}=\mathrm{a}+\mathrm{b} \cdot \mathrm{f} \\
& \mathrm{f}=\mathrm{p} \cdot(0,46 \cdot \mathrm{T}+8,13) \\
& \mathrm{a}=0,0043 \cdot \text { URmín }-\frac{\mathrm{n}}{\mathrm{N}}-1,41
\end{aligned}
$$

$$
\mathrm{b}=\mathrm{a}_{0}+\mathrm{a}_{1} \cdot \mathrm{UR}_{\text {min }}+\mathrm{a}_{2} \cdot \frac{\mathrm{n}}{\mathrm{N}}+\mathrm{a}_{3} \cdot \mathrm{u}_{2}+\mathrm{a}_{4} \cdot U R_{\text {min }} \cdot \frac{\mathrm{n}}{\mathrm{N}}+\mathrm{a}_{5} \cdot U R_{\text {min }} \cdot \mathrm{u}_{2}
$$

onde, $\mathrm{ETo}_{(\mathrm{BC})}$ é a evapotranspiração de referência $\left(\mathrm{mm} \mathrm{d}^{-1}\right)$ estimada pelo método de Blaney-Criddle (1962); URmín é a umidade relativa mínima diária (\%); n é a insolação (h) que devido a ausência de medições em campo, considerou-se a equação de Angström-Prescott para sua estimativa; N é o fotoperíodo (h); p é o fator que representa a percentagem média diária de horas de luz e varia em função da latitude e época do ano; $\mathrm{a}_{0}=0,81917 ; \mathrm{a}_{1}=-0,0040922 ; \mathrm{a}_{2}=1,0705 ; \mathrm{a}_{3}=0,065649$; $\mathrm{a}_{4}=-0,0059684 ;$ e $\mathrm{a}_{5}=-0,0005967$.

\subsection{Método de Camargo}

$$
\mathrm{ETo}_{(\mathrm{CA})}=\mathrm{R}_{\mathrm{T}} \cdot \mathrm{T} \cdot \mathrm{k}_{\mathrm{f}} \cdot \mathrm{ND}
$$

em que, $\mathrm{ETo}_{(\mathrm{CA})}$ é a evapotranspiração de referência $\left(\mathrm{mm} \mathrm{d}^{-1}\right)$ estimada pelo método de Camargo (1971); $\mathrm{R}_{\mathrm{T}}$ é a radiação solar extraterrestre ( $\mathrm{mm} \mathrm{d}^{-1}$ de evapotranspiração equivalente); ND é o número de dias do período analisado; $\mathrm{T}$ é a temperatura do ar média $\left({ }^{\circ} \mathrm{C}\right)$ e $\mathrm{k}_{\mathrm{f}}$ é um fator de ajuste que varia com a temperatura do ar média anual do local $\left(\mathrm{k}_{\mathrm{f}}=0,01\right.$ para $\mathrm{T}<23{ }^{\circ} \mathrm{C} ; \mathrm{k}_{\mathrm{f}}=0,0105$, para $\mathrm{T}=24^{\circ} \mathrm{C} ; \mathrm{k}_{\mathrm{f}}=0,011$, para $\mathrm{T}=25^{\circ} \mathrm{C} ; \mathrm{k}_{\mathrm{f}}=0,0115$, para $\mathrm{T}$ $=26^{\circ} \mathrm{C}$; e $\mathrm{k}_{\mathrm{f}}=0,012$, para $\mathrm{T}>26^{\circ} \mathrm{C}$ ).

\subsection{Método de Jensen-Haise}

$$
\mathrm{ETo}_{(\mathrm{HH})}=\mathrm{R}_{\mathrm{s}} \cdot(0,0252 \cdot \mathrm{T}+0,078)
$$

onde, $\mathrm{ETo}_{(\mathrm{JH})}$ é a evapotranspiração de referência $\left(\mathrm{mm} \mathrm{d}^{-1}\right)$ estimada pelo método de Jensen e Haise (1963) e $R_{S}$ é a radiação solar global $\left(\mathrm{mm} \mathrm{d}^{-1}\right)$.

\subsection{Método de Makkink}

$$
\begin{aligned}
& \mathrm{ETo}_{(\mathrm{MK})}=0,61 \cdot \mathrm{W} \cdot \mathrm{R}_{\mathrm{T}}-0,12 \\
& \mathrm{~W}=0,483+0,01 \cdot \mathrm{T}
\end{aligned}
$$

em que, $\mathrm{ETo}_{(\mathrm{MK})}$ é a evapotranspiração de referência $\left(\mathrm{mm} \mathrm{d}^{-1}\right)$ estimada pelo método de Makking (1957) e W é um fator de ponderação.

$\mathrm{O}$ fator $\mathrm{W}$ varia em função da temperatura do bulbo úmido, mas na falta deste dado pode-se utilizar a temperatura do ar média (Pereira et al., 1997).

\subsection{Método da radiação solar}

$$
\mathrm{ETo}_{(\mathrm{RAD})}=\mathrm{c}_{\mathrm{v}} \cdot \mathrm{W} \cdot \mathrm{R}_{\mathrm{S}}
$$

onde, $\mathrm{ETo}_{(\mathrm{RAD})}$ é a evapotranspiração de referência estimada $\left(\mathrm{mm} \mathrm{d}^{-1}\right)$ pelo método da radiação solar de 1977; W é o fator de ponderação (Equação 20) e c c é um fator de ajuste que varia 
em função dos intervalos de velocidade do vento e umidade relativa.

\subsection{Método de Araújo Filho e Cirilo}

A metodologia proposta por Araújo Filho e Cirilo (1994), desenvolvida para o estado de Pernambuco, fundamenta-se na correlação entre as variáveis climáticas e de posição geográfica com a evapotranspiração obtida através do tanque classe A. Após diversas calibrações, a equação que melhor se ajustou aos dados foi:

$$
\mathrm{ETo}_{(\mathrm{AF}-\mathrm{C})}=260+\mathrm{e}^{-1,0+0,01 \mathrm{LON}}+\mathrm{e}^{-1,0+0,19 \mathrm{~T}}-2,23 \mathrm{UR}-3,71 \ln (\mathrm{P})
$$

em que, $\mathrm{ETo}_{(\mathrm{AF}-\mathrm{C})}$ é a evapotranspiração de referência estimada pelo método de Araújo Filho e Cirilo (mm mês); LON é a longitude (graus); UR é a umidade relativa média mensal (\%) e $\mathrm{P}$ a precipitação ( $\mathrm{mm})$.

\subsection{Método de Oliveira et al.}

$$
\begin{aligned}
\mathrm{ETo}_{(\mathrm{Oli})}= & -8,0483+0,2153 \mathrm{~T}_{\text {máx }}+0,0502 \mathrm{UR}_{\text {máx }} \\
& -0,0245 \mathrm{UR}_{\text {mín }}+0,1645 \mathrm{R}
\end{aligned}
$$

em que, $\mathrm{ETo}_{(\mathrm{Oli})}$ é a evapotranspiração de referência $\left(\mathrm{mm} \mathrm{d}^{-1}\right)$ estimada pelo método de Oliveira et al. (2007).

A equação proposta por Oliveira et al. (2007) foi desenvolvida com dados da bacia do riacho da Gameleira e o coeficiente de determinação proveniente das correlações realizadas foi igual a 0,7635 . O modelo linear proposto foi comparado a métodos indiretos, tendo como variável testemunha estimativa obtida através de um lisímetro de pesagem.

\subsection{Avaliação dos métodos de estimativa da ETo}

Os dados de ETo resultantes da aplicação dos métodos apresentados foram submetidos à análise estatística. Para avaliar a precisão dos modelos, utilizou-se o coeficiente de determinação $\left(\mathrm{R}^{2}\right)$ da regressão linear entre ETo estimada por determinado método empírico e pelo método de PenmanMonteith FAO-56 (padrão).

A exatidão das estimativas, que está relacionada com o afastamento dos valores estimados através dos 11 métodos utilizados neste trabalho em relação à estimativa padrão de Penman-Monteith, foi avaliada através do índice de concordância (d) de Willmott et al. (1985), dado por:

$$
\mathrm{d}=1-\frac{\sum_{\mathrm{i}=1}^{\mathrm{N}}\left(\mathrm{P}_{\mathrm{i}}-\mathrm{O}_{\mathrm{i}}\right)^{2}}{\sum_{\mathrm{i}=1}^{\mathrm{N}}\left(\left|\mathrm{P}_{\mathrm{i}}-\mathrm{O}\right|+\left|\mathrm{O}_{\mathrm{i}}-\mathrm{O}\right|\right)^{2}}, 0 \leq \mathrm{d} \leq 1
$$

em que $\mathrm{O}_{\mathrm{i}}$ são os valores estimados pelo método padrão; $\mathrm{P}_{\mathrm{i}}$ são os valores estimados pelos métodos propostos; O é a média dos valores estimados pelo método padrão e $\mathrm{N}$ é o número de eventos.

O desempenho de cada método foi avaliado através do índice de desempenho proposto por Camargo e Sentelhas (1997), denominado de índice de concordância (c). O índice "c" reúne o coeficiente de correlação (r) e o de concordância (d), sendo expresso da seguinte forma:

$$
\mathrm{c}=\mathrm{r} . \mathrm{d}
$$

A avaliação quantitativa e qualitativa do índice de desempenho foi realizada conforme classificação listada na Tabela 1 (Camargo e Sentelhas, 1997).

$\mathrm{Na}$ quantificação dos erros proporcionados pelas estimativas, utilizaram-se os seguintes parâmetros (Gavilán et al., 2008; Back, 2008):

$$
\mathrm{CRM}=\frac{\sum_{\mathrm{i}=1}^{\mathrm{N}} \mathrm{O}_{\mathrm{i}}-\sum_{\mathrm{i}=1}^{\mathrm{N}} \mathrm{P}_{\mathrm{i}}}{\sum_{\mathrm{i}=1}^{\mathrm{N}} \mathrm{O}_{\mathrm{i}}}
$$

$$
\mathrm{EPE}=\sqrt{\left(\frac{\sum_{\mathrm{i}-1}^{\mathrm{N}}(\mathrm{Oi}-\mathrm{Pi})}{\mathrm{N}-1}\right)^{2}}
$$

em que, CRM é o coeficiente de massa residual e EPE é o erro padrão da estimativa.

Tabela 1 - Avaliação do índice de desempenho "c" proposto por Camargo e Sentelhas (1997).

\begin{tabular}{cc}
\hline Valor de "c" & Desempenho \\
\hline$>0,85$ & Ótimo \\
$0,76-0,85$ & Muito bom \\
$0,66-0,75$ & Bom \\
$0,61-0,65$ & Mediano \\
$0,51-0,6$ & Sofrível \\
$0,41-0,5$ & Mau \\
$\leq 0,40$ & Péssimo \\
\hline
\end{tabular}

\section{RESULTADOS E DISCUSSÃO}

\subsection{Métodos baseados na temperatura do ar}

Encontra-se na Figura 2 as médias mensais da ETo, obtida pelos métodos baseados na temperatura do ar.

O método de Hargreaves-Samani superestimou a ETo em relação à Penman-Monteith em todos os meses, com diferenças relativas entre $0,44 \%$ (novembro) e $8,46 \%$ (abril). É provável que essa diferença observada em abril esteja relacionada à maior nebulosidade e maior ocorrência de chuvas que se registram em abril, em relação a novembro. Esta tendência também foi verificada por Neves et al. (2005), Fernandes 


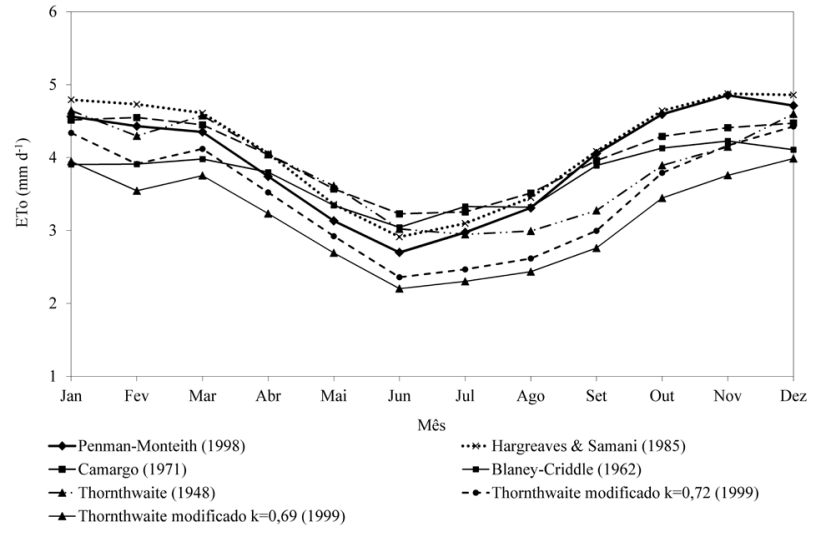

Figura 2 - Médias mensais da ETo obtida através de métodos baseados na temperatura do ar.

(2006), Araújo et al. (2007), Borges e Mediondo (2007) e Back (2008). Em relação às demais curvas apresentadas, a deste método foi a que melhor acompanhou a do método padrão de Penman-Monteith FAO-56.

O método de Camargo superestimou a ETo entre os meses de fevereiro a agosto, com diferenças relativas entre $2,25 \%$ (março) e $19,62 \%$ (junho). Nos demais meses houve subestimativa, com valores relativos entre $1,04 \%$ (janeiro) e $9,15 \%$ (novembro).

O método de Blaney-Criddle superestimou ETo nos meses de abril a agosto, com diferenças relativas variando de 0,29 a $12,81 \%$, respectivamente, e subestimou nos demais meses, com diferenças relativas variando de 4,06 a $14,41 \%$, respectivamente. $\mathrm{O}$ mesmo não foi observado por Fernandes (2006) e Back (2008), que encontraram tendência à superestimativa do método de Blaney-Criddle ao longo de todo o ano, com diferença percentual de máxima de 95 e 140\%, respectivamente.

O método de Thornthwaite superestimou a ETo nos meses de janeiro $(1,79 \%)$, março $(5,17 \%)$ a junho $(11,94 \%)$ e subestimou nos demais meses, com erro relativo de $0,94 \%$ em julho a $19,30 \%$ em setembro. Os métodos de Thornthwaite modificado com coeficiente $\mathrm{k}=0,72$, denominado de "Thorn-k1" e com coeficiente $\mathrm{k}=0,69$, denominado de "Thorn-k2", subestimaram a evapotranspiração em todos os meses do ano. O erro relativo para o método de Thorn-k1 foi de 4,92\% em janeiro e $26,15 \%$ em setembro, enquanto que, para o método de Thorn-k2 foi de 13,50\% em janeiro e 31,98\% em setembro.

Para refinar a análise dos métodos citados foi feita a análise estatística dos dados, através de coeficientes e erros de estimativa, nas escalas de tempo diária, quinquidial, quinzenal e mensal, conforme Tabela 2.

A análise da Tabela 2 permitiu verificar que dentre os seis métodos avaliados o que apresentou melhor desempenho em relação à Penman-Monteith FAO-56 foi o método de Hargreaves-Samani, que conforme a Tabela 1 apresentou desempenho "Muito bom" na escala diária e "Ótimo" nas demais escalas. Em seguida, tem-se o método de Blaney-Criddle, com desempenho "Muito bom" em todas as escalas de tempo e $\mathrm{R}^{2}$ variando de 0,796 a 0,840 . Já o método de Camargo apresentou desempenho "Muito bom", apenas nas escalas de 10, 15 e 30 dias e $\mathrm{R}^{2}$ máximo de 0,848 nesta última escala.

Para o método de Hargreaves-Samani, Gonçalves et al. (2009), Cavalcanti Junior et al. (2010), Souza et al. (2010) e Almeida et al. (2010) obtiveram, respectivamente, desempenho "Muito bom", "Muito bom", "Bom" e "Péssimo" e valores de $\mathrm{R}^{2}$ iguais a $0,65,0,75,0,87$ e 0,20 . Os resultados obtidos por estes autores foram em estudos realizados em Sobral-CE, Mossoró-RN, perímetros irrigados de Sergipe e Foratelza-CE.

Medeiros (2002), em estudo realizado em Paraipaba-CE, obteve para o método de Hargreaves e Samani desempenho "Mau" e $\mathrm{R}^{2}$ igual a 0,46, enquanto Back (2008), em estudo realizado em Urussanga-SC, obteve desempenho "Péssimo" e $\mathrm{R}^{2}$ igual a 0,94 . Observa-se que ambos não encontraram bons ajustes no emprego do método de Hargreaves-Samani e atribuíram os resultados ao fato deste ter sido desenvolvido para condições áridas, condição esta diferente das regiões estudas pelos referidos autores. O mesmo foi observado por Back (2008) ao utilizar o método de Blaney-Criddle.

Para o método de Blaney-Criddle, tanto Almeida et al. (2010), quanto Cavalcanti Junior et al. (2010) obtiveram desempenho "Ótimo" e $\mathrm{R}^{2}$ igual a 0,92 e 0,96 , respectivamente.

O método de Thornthwaite apresentou baixos coeficientes de correlação, variando de 0,41 a 0,69 , e desempenho satisfatório apenas na escala de tempo mensal, como já era esperado. O método original de Thornthwaite não estima satisfatoriamente a evapotranspiração, por não considerar o termo aerodinâmico ou contribuição da energia latente, e seu resultado pode ter sido reflexo da ausência deste termo em sua formulação (Camargo et al., 1999).

Com a introdução da temperatura efetiva, com $\mathrm{k}=0,72$, ficou evidenciado que esta modificação pode representar bem o termo aerodinâmico, resultando em estimativas mais confiáveis. Segundo Camargo et al. (1999), com a utilização da temperatura efetiva e $\mathrm{k}=0,72$, o desempenho do método de Thornthwaite melhorou significativamente, conforme evidenciado neste estudo. Os resultados encontrados por Medeiros (2002) e Back (2008), indicaram o mesmo padrão. Já o método modificado com $\mathrm{k}=0,69$, foi pouco satisfatório, com desempenho ruim em todas as escalas de tempo analisadas.

Pereira e Pruitt (2004), embora tenham analisado a equação de Thornthwaite modificada na escala diária, concluíram que com a utilização da temperatura efetiva, o desempenho encontrado, em relação às estimativas de um 
Tabela 2 - Análise estatística da correlação entre os métodos baseados na temperatura e o método de Penman-Monteith FAO-56 nas escalas de tempo diária, quinquidial, decendial, quinzenal e mensal.

\begin{tabular}{|c|c|c|c|c|c|c|c|c|}
\hline \multirow{2}{*}{ Método } & \multirow{2}{*}{$\begin{array}{c}\text { Escala } \\
\text { (dias) }\end{array}$} & \multicolumn{4}{|c|}{ Índices } & \multicolumn{2}{|c|}{ Erros $\left(\mathrm{mm} \mathrm{d}^{-1}\right)$} & \multirow{2}{*}{$\begin{array}{l}\mathrm{N}^{\circ} \mathrm{de} \\
\text { dados }\end{array}$} \\
\hline & & $\mathrm{R}^{2}$ & $\mathrm{~d}$ & $\mathrm{c}$ & Desempenho & EPE & CRM & \\
\hline \multirow{5}{*}{$\begin{array}{l}\text { Hargreaves } \\
\text { \& Samani } \\
(1985)\end{array}$} & 1 & 0,753 & 0,911 & 0,79 & Muito Bom & 0,521 & $-0,046$ & 1700 \\
\hline & 5 & 0,861 & 0,947 & 0,88 & Ótimo & 0,361 & $-0,044$ & 355 \\
\hline & 10 & 0,896 & 0,957 & 0,91 & Ótimo & 0,314 & $-0,045$ & 180 \\
\hline & 15 & 0,911 & 0,962 & 0,92 & Ótimo & 0,297 & $-0,046$ & 122 \\
\hline & 30 & 0,937 & 0,968 & 0,94 & Ótimo & 0,262 & $-0,046$ & 64 \\
\hline \multirow{5}{*}{$\begin{array}{l}\text { Camargo } \\
\text { (1971) }\end{array}$} & 1 & 0,577 & 0,762 & 0,58 & Sofrível & 0,689 & $-0,015$ & 1700 \\
\hline & 5 & 0,707 & 0,845 & 0,71 & Bom & 0,504 & $-0,015$ & 355 \\
\hline & 10 & 0,765 & 0,877 & 0,77 & Muito Bom & 0,439 & $-0,017$ & 180 \\
\hline & 15 & 0,792 & 0,889 & 0,79 & Muito Bom & 0,415 & $-0,018$ & 122 \\
\hline & 30 & 0,848 & 0,912 & 0,84 & Muito Bom & 0,363 & $-0,019$ & 64 \\
\hline \multirow{5}{*}{$\begin{array}{l}\text { Blaney- } \\
\text { Criddle } \\
(1962)\end{array}$} & 1 & 0,815 & 0,933 & 0,84 & Muito Bom & 0,482 & 0,056 & 1700 \\
\hline & 5 & 0,796 & 0,903 & 0,81 & Muito Bom & 0,454 & 0,056 & 355 \\
\hline & 10 & 0,801 & 0,889 & 0,80 & Muito Bom & 0,446 & 0,056 & 180 \\
\hline & 15 & 0,808 & 0,885 & 0,80 & Muito Bom & 0,442 & 0,055 & 122 \\
\hline & 30 & 0,840 & 0,873 & 0,80 & Muito Bom & 0,432 & 0,054 & 64 \\
\hline \multirow{5}{*}{$\begin{array}{c}\text { Thornthwaite } \\
\text { (1948) }\end{array}$} & 1 & 0,345 & 0,699 & 0,41 & Mau & 0,804 & 0,021 & 1700 \\
\hline & 5 & 0,495 & 0,805 & 0,57 & Sofrível & 0,607 & 0,025 & 355 \\
\hline & 10 & 0,555 & 0,840 & 0,63 & Mediano & 0,540 & 0,024 & 180 \\
\hline & 15 & 0,577 & 0,851 & 0,65 & Mediano & 0,521 & 0,023 & 122 \\
\hline & 30 & 0,626 & 0,874 & 0,69 & Bom & 0,475 & 0,022 & 64 \\
\hline \multirow{5}{*}{$\begin{array}{c}\text { Thornthwaite } \\
\text { modificado } \\
\mathrm{k}=0,72 \\
(1999)\end{array}$} & 1 & 0,448 & 0,731 & 0,49 & Mau & 0,865 & 0,113 & 1700 \\
\hline & 5 & 0,640 & 0,810 & 0,65 & Mediano & 0,693 & 0,116 & 355 \\
\hline & 10 & 0,714 & 0,836 & 0,71 & Bom & 0,635 & 0,115 & 180 \\
\hline & 15 & 0,740 & 0,844 & 0,73 & Bom & 0,619 & 0,116 & 122 \\
\hline & 30 & 0,807 & 0,863 & 0,77 & Muito Bom & 0,577 & 0,116 & 64 \\
\hline \multirow{5}{*}{$\begin{array}{c}\text { Thornthwaite } \\
\text { modificado } \\
\mathrm{k}=0,69 \\
(1999)\end{array}$} & 1 & 0,425 & 0,604 & 0,39 & Péssimo & 1,066 & 0,190 & 1700 \\
\hline & 5 & 0,608 & 0,666 & 0,52 & Sofrível & 0,936 & 0,193 & 355 \\
\hline & 10 & 0,680 & 0,688 & 0,57 & Sofrível & 0,893 & 0,192 & 180 \\
\hline & 15 & 0,705 & 0,696 & 0,58 & Sofrível & 0,879 & 0,192 & 122 \\
\hline & 30 & 0,770 & 0,712 & 0,63 & Mediano & 0,850 & 0,192 & 64 \\
\hline
\end{tabular}

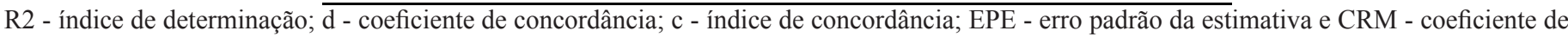
massa residual.

lisímetro de pesagem, foi similar ao desempenho encontrado através da equação de Penman-Monteith FAO-56. O estudo realizado pelos referidos autores foi conduzido em duas áreas distintas, uma com clima semiárido e outra com clima úmido, utilizando a equação de Thornthwaite modificada com $\mathrm{k}=0,69$.

Quanto a análise dos erros, observa-se na Tabela 2, que o método de Hargreaves-Samani foi o que apresentou menor erro padrão da estimativa $\left(0,26 \mathrm{~mm} \mathrm{dia}^{-1}\right)$, e o que apresentou maior erro foi o método de Thornthwaite modificado com $\mathrm{k}=$ 0,69 , com erro padrão da estimativa igual a $1,1 \mathrm{~mm} \mathrm{dia}^{-1}$.

Os resultados dos métodos baseados na temperatura refletiram as semelhanças e diferenças climáticas existentes entre o local estudado neste trabalho, semiárido, e o local onde estas equações foram desenvolvidas, para condições áridas. Daí se justifica os bons resultados obtidos pelos métodos de Hargreaves-Samani e Blaney-Criddle.

\subsection{Métodos baseados na radiação solar}

Na Figura 3 são mostrados os resultados dos métodos baseados na radiação solar. Observou-se que os métodos de

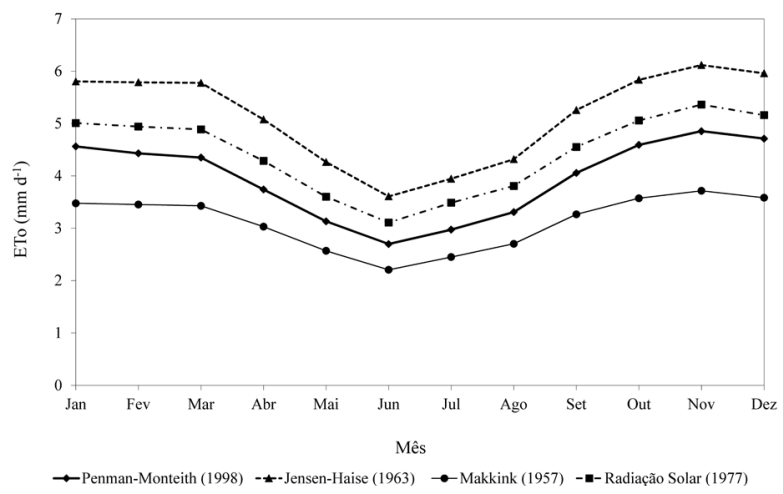

Figura 3 - Médias mensais da ETo obtida através de métodos baseados na radiação solar. 
Tabela 3 - Análise estatística da correlação entre os métodos baseados na radiação solar e o método de Penman-Monteith FAO-56 nas escalas de tempo diária, quinquidial, decendial, quinzenal e mensal.

\begin{tabular}{|c|c|c|c|c|c|c|c|c|}
\hline \multirow{2}{*}{ Método } & \multirow{2}{*}{$\begin{array}{c}\text { Escala } \\
\text { (dias) }\end{array}$} & \multicolumn{4}{|c|}{ Índices } & \multicolumn{2}{|c|}{ Erros $\left(\mathrm{mm} \mathrm{d}^{-1}\right)$} & \multirow{2}{*}{$\begin{array}{l}\mathrm{N}^{\circ} \mathrm{de} \\
\text { dados }\end{array}$} \\
\hline & & $\mathrm{R}^{2}$ & $\mathrm{~d}$ & $\mathrm{c}$ & Desempenho & EPE & CRM & \\
\hline \multirow{5}{*}{$\begin{array}{c}\text { Jensen- } \\
\text { Haise (1963) }\end{array}$} & 1 & 0,945 & 0,756 & 0,73 & Bom & 1,289 & $-0,302$ & 1730 \\
\hline & 5 & 0,950 & 0,684 & 0,67 & Bom & 1,259 & $-0,304$ & 361 \\
\hline & 10 & 0,954 & 0,658 & 0,64 & Mediano & 1,248 & $-0,304$ & 183 \\
\hline & 15 & 0,957 & 0,654 & 0,64 & Mediano & 1,240 & $-0,304$ & 124 \\
\hline & 30 & 0,963 & 0,637 & 0,63 & Mediano & 1,227 & $-0,304$ & 64 \\
\hline \multirow{5}{*}{$\begin{array}{l}\text { Makkink } \\
\text { (1957) }\end{array}$} & 1 & 0,933 & 0,791 & 0,76 & Muito Bom & 0,898 & 0,211 & 1730 \\
\hline & 5 & 0,941 & 0,717 & 0,70 & Bom & 0,892 & 0,211 & 361 \\
\hline & 10 & 0,948 & 0,688 & 0,67 & Bom & 0,890 & 0,211 & 183 \\
\hline & 15 & 0,952 & 0,681 & 0,66 & Bom & 0,889 & 0,211 & 124 \\
\hline & 30 & 0,963 & 0,667 & 0,65 & Mediano & 0,875 & 0,210 & 64 \\
\hline \multirow{5}{*}{$\begin{array}{c}\text { Radiação } \\
\text { Solar (1977) }\end{array}$} & 1 & 0,942 & 0,929 & 0,90 & Ótimo & 0,589 & $-0,123$ & 1730 \\
\hline & 5 & 0,952 & 0,912 & 0,89 & Ótimo & 0,541 & $-0,124$ & 361 \\
\hline & 10 & 0,960 & 0,905 & 0,89 & Ótimo & 0,524 & $-0,123$ & 183 \\
\hline & 15 & 0,963 & 0,903 & 0,89 & Ótimo & 0,520 & $-0,123$ & 124 \\
\hline & 30 & 0,971 & 0,898 & 0,88 & Ótimo & 0,511 & $-0,124$ & 64 \\
\hline
\end{tabular}

R2 - índice de determinação; d - coeficiente de concordância; c - índice de concordância; EPE-erro padrão da estimativa e CRM-coeficiente de massa residual.

Jensen-Haise e radiação solar superestimaram ETo em todos os meses, com diferenças relativas entre entre $26,04 \%$ (novembro) e $36,18 \%$ (maio), para o primeiro método, e $9,53 \%$ (dezembro) e $17,27 \%$ (julho), para o segundo método citado.

Já o método de Makkink apresentou padrão diferente, subestimando a ETo em todos os meses do ano, com valores entre 17,59\% (julho) a 23,97\% (dezembro). Os mesmos padrões foram observados por Vescove e Turco (2005) e Fernandes (2006). Estas diferenças foram superiores às observadas para os métodos de HargreavesSamani, Camargo e Blaney-Criddle na escala mensal.

A Tabela 3 resume os índices estatísticos e erros provenientes das correlações realizadas com os métodos baseados na radiação. Observou-se que o método da Radiação Solar foi o que apresentou melhor correlação em relação à PenmanMonteith FAO-56, com $\mathrm{R}^{2}$ igual a 0,971 na escala mensal. $\mathrm{O}$ mesmo foi observado por Mendonça e Dantas (2010), em estudo realizado em Capim-PB $\left(\mathrm{R}^{2}=0,907\right)$ e Oliveira et al. (2008), em estudo realizado na mesma área desta pesquisa $\left(R^{2}=0,706\right)$.

O método de Makkink apresentou bom desempenho nas escalas de 1 a 15 dias. Já no método Jensen-Haise, o desempenho foi satisfatório apenas nas escalas de 1 e 10 dias. Em ambos os métodos, observou-se que o desempenho não acompanhou o aumento da escala de tempo.

O método da radiação solar é uma adaptação do método de Jensen-Haise e as alterações oriundas desta adaptação podem ter contribuído para seu melhor desempenho. Há de se considerar também que nas formulações dos métodos de Jensen-Haise e da radiação solar utiliza-se a radiação solar global, e na formulação do método de Makkink utiliza-se a radiação extraterrestre. $\mathrm{Na}$ formulação do método da radiação solar também é considerado um fator de ponderação, determinado em função da velocidade do vento e umidade relativa do ar.

Analisando diversos trabalhos realizados sobre evapotranspiração, como Vescove e Turco (2005), Fernandes (2006), Gonçalves et al. (2009), Almeida et al. (2010) e Cavalcanti Junior et al. (2010), observou-se que os métodos baseados na radiação apresentaram bons resultados em todos eles. Este padrão indica que os métodos baseados na radiação solar independem das condições climáticas da área estudada, diferentemente do que ocorre com os métodos baseados na temperatura do ar.

\subsection{Métodos baseados em modelagem estatística}

A Figura 4 ilustra os resultados dos métodos baseados em modelagem estatística. Como se observa, o métodos de Araújo

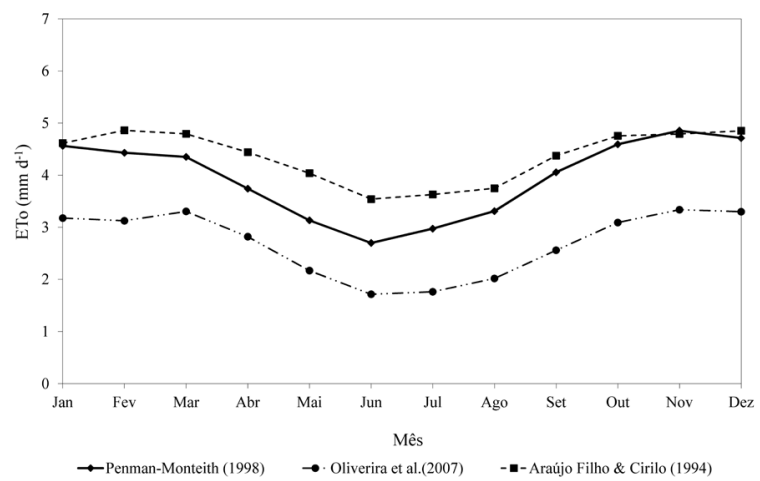

Figura 4 - Médias mensais da evapotranspiração de referência (ETo) obtida pelos métodos baseados em modelagem estatística. 
Tabela 4 - Análise estatística da correlação entre os métodos baseados em modelagem estatística e o método de Penman-Monteith FAO-56 nas escalas de tempo diária, quinquidial, decendial, quinzenal e mensal.

\begin{tabular}{|c|c|c|c|c|c|c|c|c|}
\hline \multirow{2}{*}{ Método } & \multirow{2}{*}{$\begin{array}{c}\text { Escala } \\
\text { (dias) }\end{array}$} & \multicolumn{4}{|c|}{ Índices } & \multicolumn{2}{|c|}{ Erros $\left(\mathrm{mm} \mathrm{d}^{-1}\right)$} & \multirow{2}{*}{$\begin{array}{l}\mathrm{N}^{\circ} \text { de } \\
\text { dados }\end{array}$} \\
\hline & & $\mathrm{R}^{2}$ & $\mathrm{~d}$ & $\mathrm{c}$ & Desempenho & EPE & CRM & \\
\hline \multirow{5}{*}{$\begin{array}{l}\text { Oliveira et al. } \\
\quad(2007)\end{array}$} & 1 & 0,886 & 0,632 & 0,59 & Sofrível & 1,324 & 0,315 & 1550 \\
\hline & 5 & 0,876 & 0,559 & 0,52 & Sofrível & 1,302 & 0,312 & 324 \\
\hline & 10 & 0,883 & 0,539 & 0,51 & Sofrível & 1,294 & 0,311 & 164 \\
\hline & 15 & 0,886 & 0,533 & 0,50 & Mau & 1,292 & 0,311 & 111 \\
\hline & 30 & 0,878 & 0,513 & 0,48 & Mau & 1,293 & 0,310 & 56 \\
\hline $\begin{array}{l}\text { Araújo Filho e } \\
\text { Cirilo (1994) }\end{array}$ & 30 & 0,499 & 0,779 & 0,55 & Sofrível & 0,671 & $-0,087$ & 63 \\
\hline
\end{tabular}

R2 - índice de determinação; $\overline{\mathrm{d} \text { - coeficiente de concordância; c - índice de concordância; EPE - erro padrão da estimativa }}$ e CRM - coeficiente de massa residual.

Filho e Cirilo (1994) e Oliveira et al. (2007) apresentaram padrão similar ao de Penman-Monteith FAO-56, com valores mínimos da ETo correspondentes ao período do meio do ano.

O método de Oliveira et al. (2007) subestimou a ETo em todos os meses do ano, com diferença relativa variando entre $24,06 \%$ (março) a 40,76\% (julho). Com padrão contrário ao observado anteriormente, o método de Araújo Filho e Cirilo (1994), com exceção do mês de novembro, superestimou a ETo em todos os meses do ano, com diferenças entre $1,15 \%$ (janeiro) e $31,21 \%$ (junho).

Mesmo apresentando tendências similares ao método de Penman-Monteith FAO-56, os métodos baseados em modelagem estatística não se ajustaram adequadamente. Como se observa na Figura 4, a diferença entre os valores estimados e o padrão variou, refletindo diretamente no desempenho e na precisão destes métodos, principalmente no método de Araújo Filho e Cirilo (1994), como demonstram os resultados da análise estatística na Tabela 4.

O método de Oliveira et al. (2007) apresentou elevados coeficientes de determinação, variando de 0,876 a 0,886. Entretanto, o desempenho não foi satisfatório em nenhuma escala de tempo. Os resultados mostram que o grau de dispersão entre os dados não foi grande, entretanto, o afastamento entre eles foi significativo, refletindo no desempenho.

A correlação entre Penman-Monteith FAO-56 e o método de Araújo Filho e Cirilo (1994) resultou em baixo coeficiente de correlação $\left(\mathrm{R}^{2}=0,499\right)$, e desempenho "Sofrível". Estes resultados indicam que tanto a dispersão, quanto o afastamento entre os dados, foram relevantes.

Quanto aos métodos analisados, baseados em modelagem estatística, destaca-se que o método de Araújo Filho e Cirilo (1994) foi obtido através de correlações de umidade relativa, precipitação e posição geográfica. As variáveis consideradas em sua formulação podem não ser adequadas para a área de estudo considerada, não estimando satisfatoriamente a evapotranspiração. Já o método de Oliveira et al. (2007) correlaciona dados de temperatura do ar, umidade relativa, velocidade do vento e radiação solar. Embora seja teoricamente mais completo que o método anterior, seus resultados também não foram satisfatórios.

\section{CONCLUSÕES}

Através dos resultados obtidos no presente estudo, dadas às condições climáticas da área estudada, pode-se chegar às seguintes conclusões:

1. Dentre os métodos baseados na temperatura o método de Hargreaves-Samani é o que apresentou melhores estimativas da evapotranspiração de referência.

2. A utilização da temperatura efetiva $(\operatorname{com~} k=0,72)$ na equação original de Thornthwaite melhora significativamente o desempenho deste método.

3. Dentre os métodos baseados na radiação solar, o que apresenta melhores estimativas é o método da radiação solar.

4. Os métodos baseados em modelagem estatística não apresentam bom desempenho nas escalas de tempo analisadas.

\section{AGRADECIMENTOS}

Os autores agradecem à UFPE e ao Instituto Federal de Educação, Ciência e Tecnologia, campus de Vitória de Santo Antão - PE (IFPE Vitória), pela colaboração técnica; à FINEP/ CT- HIDRO pelo apoio financeiro e ao CNPq/CT-HIDRO pelas bolsas de Produtividade em Pesquisa, mestrado e doutorado, e pelas contribuições da equipe de revisores deste artigo.

\section{REFERÊNCIAS BIBLIOGRÁFICAS}

ALLEN, R.G., PEREIRA, L. S., RAES, D., SMITH, M. Crop evapotranspiration: guidelines of computing crop water 
requirements. FAO. Irrigation and Drainage Paper, 56, Roma, p.300, 1998.

ALMEIDA, B. M. et al. Comparação de métodos de estimativa da ETo na escala mensal em Fortaleza-CE. Revista Brasileira de Agricultura Irrigada, Fortaleza-CE, v.4, n.2, p.93-98, 2010.

ARAÚJO FILHO, P. F.; CIRILO, J. A. Regionalização da evapotranspiração para o estado de Pernambuco. In: Simpósio Brasileiro de Rec. Hídricos, Fortaleza-CE, Anais, 1994. CD Rom.

ARAÚJO FILHO, P. F., CABRAL, J. J. S. P., AZEVEDO, J. R. G. Considerações sobre a variabilidade espacial da precipitação na bacia do riacho Gameleira em Pernambuco. In: Simpósio Brasileiro de Recursos Hídricos, São Paulo-SP. Anais, 2007. CD Rom.

ASSAD, E. D.; PINTO, H. S. (Coordenador). Aquecimento Global e Cenários Futuros da Agricultura Brasileira. São Paulo: EMBRAPA, 2008. 84p.

BACK, A. J. Desempenho de métodos empíricos baseados na temperatura do ar para a estimativa da evapotranspiração de referência em Urussanga. Irriga, Botucatu-SP, v.13, n.4, p.449-466, 2008.

BLANEY H. F., CRIDDLE W. D. Determining consumptive use and irrigation water requirements. USDA Technical Bulletin 1275, US Department of Agriculture, Beltsville, 1962.

BORGES, A.C.; MEDIONDO, E. M. Comparação entre equações empíricas para estimativa da evapotranspiração de referência na bacia Rio Jacupiranga, SP. Revista Brasileira de Engenharia Agrícola e Ambiental, Campina GrandePB, v. 11, n. 3, p.299-300, 2007.

BRAGA, R. A. P. Gestão ambiental da bacia do rio Tapacurá

- Plano de Ação. Universidade Federal de Pernambuco/ CTG/DECIVIL/GRH. Recife-PE, 2001. 101p.

CAMARGO, A.P. de. Balanço hídrico no Estado de São Paulo. Campinas: Instituto Agronômico, 24p. Boletim 116, 1971.

CAMARGo, A.P.; SENTElhas, P. C. Avaliação do desempenho de diferentes métodos de estimativa da evapotranspiração potencial no estado de São Paulo, Brasil. Revista Brasileira de Agrometeorologia, Santa Maria, v. 5, n. 1, p.89-97, 1997.

CAMARGO, A.P. et al. Ajuste da equação de Thornthwaite para estimar a evapotranspiração potencial em climas áridos e superúmidos, com base na amplitude térmica diária. Revista Brasileira de Agrometeorologia, Santa Maria, v. 7, n. 2, p.251-257, 1999.

CAVALCANTI JUNIOR, E.G. et al. Estimativa da evapotranspiração de referência para a cidade de MossoróRN. Revista Brasileira de Agricultura Irrigada, FortalezaCE, v.4, n.2, p.87-92, 2010.
CONCEIÇÃO, M. A. F.; MANDELLI, F. Comparação entre métodos de estimativa da evapotranspiração de referência em bento Gonçalves, RS. Revista Brasileira de Agrometeorologia, Santa Maria, v. 13, n. 2, p. 303-307, 2005.

FERNANDES, L. C. Avaliação de diversas equações empíricas de evapotranspiração. Estudo de caso: Campos dos Goytacazes e Ilha do Fundão-RJ. 2006. 142f. Tese (Doutorado) - Universidade Federal do Rio de Janeiro, 2006.

FERNANDES, D. S., HEINEMANN, A. B., PAZ, R. L. F., AMORIM, A. O. Calibração regional e local da equação de Hargreaves para estimativa da evapotranspiração de referência. Revista Ciência Agronômica, v.43, n.2, p.246255, 2012.

GAVILÁN, P., ESTEVEZ, J.; BERENGENA, J. Comparison of standardized reference evapotranspiration equations in Southern Spain. Journal of Irrigation and Drainage Engineering, v. 134, n. 1, 2008.

GONÇALVES, F. M. et al. Comparação de métodos da estimativa da evapotranspiração de referência para o município de Sobral - CE. Revista Brasileira de Agricultura Irrigada, Fortaleza-CE, v.3, n.2, p.71-77, 2009.

HARGREAVES, G.H.; SAMANI, Z. Reference crop evapotranspiration from temperature. Journal of Applied Engineering in Agriculture, St Joseph, v.1, n.2, p.96-99, 1985.

JENSEN, M.E.; HAISE, H.R. Estimating evapotranspiration from solar radiation. Journal of Irrigation Drainage Division, ASCE, New York, v.89, n.1, p.15-41, 1963.

MAKKINK, G, F. Ekzamento de la formulo de Penman. Netherlands Journal of Agricultural Science, Wageningen, v. 5, p. 290-305, 1957.

MEDEIROS, A. T. Estimativa da evapotranspiração de referência a partir da equação de Penman-Monteith, de medidas lisimétricas e de equações empíricas em Paraíba, CE. 2002. 95f. Tese (Doutorado) - Escola Superior de Agricultura Luiz de Queiroz: Universidade de São Paulo, 2002.

MENDONÇA, E. A.; DANTAS, R. T. Estimativa da evapotranspiração de referência no município de Capim, $\mathrm{PB}$. Revista Brasileira de Engenharia Agrícola e Ambiental, Campina Grande-PB, v.14, n.2, p.196-202, 2010.

NEVES, F. F., SILVA, F. G. B.; CRESTANA, S. Comparação entre três métodos de evapotranspiração potencial aplicados a duas sub-bacias hidrográficas de Descalvado - SP. In: Simpósio Brasileiro de Recursos Hídricos, 2005, João Pessoa. Anais, 2005. CD Rom.

OLIVEIRA, L. M. M. et al. Modelos de regressão linear e não linear usando variáveis meteorológicas locais na estimativa 
da evapotranspiração de referência (ETo), comparados com métodos indiretos, na bacia experimental do riacho Gameleira. In: Simpósio Brasileiro de Recursos Hídricos, 2007, São Paulo. Anais, CD Rom.

OLIVEIRA, L. M. M. et al. Evapotranspiração de referência na bacia experimental do riacho Gameleira, PE, utilizandose lisímetro e métodos indiretos. Revista Brasileira de Ciências Agrárias, Recife, v.3, n.1, p.58-67, 2008.

PEREIRA, A. R.; VILLA NOVA, N. A.; SEDIYAMA, G. C. Evapo(transpi)ração. Piracicaba: FEALQ, 1997. 183p.

PEREIRA, A.R., PRUITT, W.O. Adaptation of the Thornthwaite scheme for estimating daily reference evapotranspiration. Agricultural Water Management, Amsterdam, Netherlands, v. 66, n. 2, p. 251-257, 2004.

SOUSA, I. F. et al. Evapotranspiração de referência nos perímetros irrigados do estado de Sergipe. Revista Brasileira de Engenharia Agrícola e Ambiental, Campina Grande-PB, v.14, n.6, p.633-644, 2010.
THORNTHWAITE, C.W. Na approach toward a rational classification of climate. Geographical Review, 38: 5594, 1948.

TURCO, J. E. P.; PERECIN, D.; PINTO JÚNIOR, D. L. P. Influência da acurácia de instrumentos de medidas na comparação de métodos de estimativa da evapotranspiração de referência. Botucatu, SP. Irriga, v.13, n.1, p.63-80, 2008.

VAREJÃO-SILVA, M. A. Meteorologia e Climatologia. Recife: 2006. Versão Digital, 443p.

VESCOVE, H. V.; TURCO, J. E. P. Comparação de três métodos de estimativa da evapotranspiração de referência para a região de Araraquara-SP. Revista Engenharia Agrícola, Jaboticabal-SP, v.25, n.3, p.713-721, 2005.

WILLMOTT, C.J. et al. Statistics for the evaluation and comparison of models. Journal of Geophysical Research, Ottawa, v. 90, n. 5, p.8995-9005, 1985. 